\title{
Formación de profesores en competencias TIC para dinamizar las prácticas pedagógicas en básica primaria
}

\section{Teacher training in ict skills to stimulate pedagogical practices in elementary school}

\author{
GUTIÉRREZ, Excelsa P. ${ }^{1}$ \\ ALFONSO, Nohora E. ${ }^{2}$ \\ CEPEDA, Carmen H. ${ }^{3}$ \\ CUCUNUBÁ, Yaneth ${ }^{4}$
}

\begin{abstract}
Resumen
Este artículo presenta el resultado de una propuesta para la formación de profesores de básica primaria en competencias TIC, con el propósito de fortalecer estas competencias y dinamizar las prácticas pedagógicas. El trabajo se desarrolló bajo el enfoque de investigación cualitativa y privilegia la investigación Acción Pedagógica; los resultados confirman que reflexionar las prácticas pedagógicas colectivamente enriquece el quehacer pedagógico, así mismo la implementación de las TIC en el aula genera cambios metodológicos en los procesos de enseñanza y aprendizaje.

Palabras clave: formación de profesores, práctica pedagógica, tecnologías de la información y la comunicación (TIC), saber pedagógico.
\end{abstract}

\begin{abstract}
This article presents the result of a proposal for the training of elementary school teachers in ICT competencies, with the purpose of strengthening these competencies and stimulating pedagogical practices. The work was developed under the qualitative research approach and privileges the Pedagogical Action research; The results confirm that reflecting on pedagogical practices collectively enriches the pedagogical work, likewise the implementation of ICT in the classroom generates methodological changes in the teaching and learning processes.

Keywords: teacher training, pedagogical practice, technology of the information and communication, pedagogical knowledge
\end{abstract}

\section{Introducción}

Las competencias en Tecnologías de Información y Comunicación (TIC) en la actualidad se han convertido en una necesidad para los profesionales de las diferentes disciplinas y por supuesto para los profesionales en educación debe ser necesario, ya que se están formando generaciones totalmente digitales y con una visión competitiva

\footnotetext{
${ }^{1}$ Investigadora Grupo de Investigación para la Animación Cultural Muisuata, estudiante de Maestría en TIC Aplicadas a las Ciencias de la Educación, Universidad Pedagógica y Tecnológica de Colombia, Duitama, Colombia. pilar.gutierrez436@gmail.com

2 Investigadora y directora Grupo de Investigación para la Animación Cultural Muisuata. Universidad Pedagógica y Tecnológica de Colombia, Duitama, Colombia.nohora.alfonso@uptc.edu.co

${ }^{3}$ Investigadora Grupo de investigación GIE. Universidad Pedagógica y Tecnológica de Colombia, Duitama, Colombia. carmen.cepeda@uptc.edu.co ${ }^{4}$ Investigadora Grupo de Investigación para la Animación Cultural Muisuata. Universidad Pedagógica y Tecnológica de Colombia, Duitama, Colombia. Email: yaneth.cucunuba@uptc.edu.co
} 
que el mundo actual exige, como lo menciona la Organización de las Naciones Unidas para la Educación, la Ciencia y la Cultura (UNESCO) (2008), "es fundamental que todos los docentes estén preparados para ofrecer esas oportunidades a sus estudiantes" (p.2). Por lo tanto, se hace necesaria la formación de profesores en competencias TIC para integrarlas en los procesos de enseñanza, de allí la importancia de que esta se ofrezca desde las instituciones de educación superior donde cada uno de los futuros profesores inician su formación.

No obstante, es importante resaltar la pertinencia de los procesos de formación de profesores en ejercicio de tal forma que estén en actualización continua para apropiar las competencias TIC que presenta la sociedad actual y de esta forma integrarlas a los procesos de enseñanza y aprendizaje. Domingo y Marquès (2011) manifiestan que "en el ámbito educativo, las TIC pueden proporcionar un entorno de enseñanza y aprendizaje para el alumnado y profesorado" (p.170); a su vez, Cabero (2010) resalta que "las TIC son medios significativos para el aprendizaje, entornos de innovación escolar, y para la comunicación e interacción social" (p.39); por ende, es fundamental que los profesores apropien dichas competencias que les permitan innovar en el aula.

El proceso de investigación que se presenta en este artículo se soportó en tres conceptos fundantes referentes a la formación de profesores, la práctica pedagógica y las competencias TIC para profesores de básica primaria. La investigación se llevó a cabo con el fin de fortalecer las competencias digitales en un grupo de profesores de básica primaria pertenecientes a la Institución Educativa Técnica Agrícola (IETA) de Paipa Boyacá en Colombia.

El desarrollo del proceso se implementó en tres momentos: la reflexión con los profesores acerca de las prácticas pedagógicas mediadas por las TIC con el fin de identificar fortalezas y debilidades sobre las mismas; la propuesta de práctica pedagógica renovadora por medio de un programa de formación de profesores en TIC para dinamizar las prácticas pedagógicas y, finalmente la valoración de la práctica pedagógica implementada, a través de la satisfacción personal de profesores frente a los cambios observados, fundamentados en la revisión del plan de área y diarios de campo.

Esta investigación cobró mayor relevancia ya que a partir de la emergencia sanitaria causada por el COVID- 19 y las medidas de prevención adoptadas por el gobierno nacional, los estudiantes que recibían sus clases en forma presencial tuvieron que hacerlo desde sus casas, dando paso a la educación virtual, llevando a que el uso de las TIC pasará a convertirse en una herramienta fundamental en el campo de la educación, donde los profesores tuvieron que reconsiderar sus estrategias de enseñanza y replantear la forma para llegar a cada uno de sus estudiantes hasta los lugares más apartados.

Algunos profesores se vieron en dificultades para cumplir con las nuevas exigencias educativas ya que sus habilidades en el manejo de herramientas tecnológicas son muy básicas. De igual manera hubo que reinventarse una forma de llegar a sus estudiantes teniendo en cuenta que su trabajo se desarrolla en sectores rurales donde hay carencia de conectividad, familias que no cuentan con dispositivos TIC y que desconocen como brindar orientación y acompañamiento a sus hijos e hijas.

\subsection{Formación de profesores y prácticas pedagógicas}

En relación a la formación de profesores, esta se viene desarrollando desde las diferentes entidades preocupadas por la educación a nivel mundial, partiendo desde la formación inicial y continuando con la formación para los profesores que están en ejercicio, "la formación del profesorado ha de conducir a una adquisición (en el caso de los profesores en formación) o bien a un perfeccionamiento, enriquecimiento de la competencia profesional de los docentes que se implican en tareas de formación" (Marcelo, 1994, cap. I). De igual manera, es importante resaltar que la formación de profesores permite a partir de un trabajo colaborativo, fortalecer las competencias disciplinares y profesionales de estos, de tal forma que se vean inmersos en la transformación de sus prácticas pedagógicas en el aula. 
Para el caso de Colombia, el Ministerio de Educación Nacional (MEN) a través del Plan de Desarrollo 2010-2014 del gobierno presidencial de Juan Manuel Santos, con su programa Prosperidad para Todos, implementó una política de "Formación docente para la calidad educativa" esto con el fin de poner en práctica acciones y desarrollar proyectos de formación con docentes y directivos docentes de tal forma que se lograra mejorar la calidad de la educación preescolar, básica y media. Esta política educativa fue orientada al fortalecimiento de la formación inicial de docentes y a la cualificación, actualización y perfeccionamiento de los educadores en servicio. Así mismo, el MEN y las secretarías de educación, hicieron acompañamiento de modo que se lograra consolidar la estrategia de formación.

Históricamente los procesos de formación de profesores en Colombia se han orientado a mejorar la calidad educativa partiendo desde los profesores, de tal forma que sean ellos quienes, a partir de la innovación en sus prácticas pedagógicas, logren esos cambios significativos que requiere la educación. El concepto de práctica pedagógica que se expone a continuación está fundamentado en varios autores y se enfoca principalmente en la actividad que los profesores desarrollan a diario en sus aulas con sus estudiantes, permitiendo así enriquecer el saber pedagógico a partir de la interacción recíproca. EI MEN de Colombia define la práctica pedagógica como "Un proceso de auto reflexión, que se convierte en el espacio de conceptualización, investigación y experimentación didáctica" (Ministerio de Educación Nacional, 2019, p.5)

Por su parte, Quero (2006) define la práctica pedagógica como "la actividad diaria que desarrollamos en las aulas, laboratorios u otros espacios, orientada por un currículo y que tiene como propósito la formación de nuestros alumnos" (p.90); de la misma forma, Danielson (2013) define la práctica pedagógica como aquella que permite a los profesores reflexionar sobre "cómo planea, cómo enseña, cómo se comunica, cómo crea ambientes amables y respetuosos, cómo usa la evaluación para el crecimiento de sus estudiantes entre otros aspectos" (p.3). Wilson (citado por Castillo, 2008) concibe la práctica pedagógica como "el conjunto de actividades que permiten planificar, desarrollar y evaluar procesos intencionados de enseñanza mediante los cuales se favorece el aprendizaje de contenidos conocimientos, habilidades, actitudes y valores (p.179), por otra parte, Davini (2008) entiende a las prácticas docentes como el "campo de aplicación de conocimientos, métodos y técnicas para enseñar" (p.5).

A partir de los conceptos dados por los autores referenciados, se puede decir que la práctica pedagógica es el quehacer diario que los profesores desarrollan desde el aula como facilitadores y orientadores del conocimiento, es a través del saber pedagógico que se fortalecen las prácticas pedagógicas, y de esa forma se enriquecen los procesos de enseñanza. Reflexionar la práctica pedagógica permite a los profesores hacer una revisión sólida desde la planeación, metodologías y estrategias didácticas, logrando no sólo el aprendizaje en conocimientos sino también en la concepción de ser humano que se quiere formar.

\subsection{Formación en competencias TIC para profesores de básica primaria}

Es importante recalcar que las TIC han generado un gran impacto en el campo educativo, donde los profesores de esta época enfrentan cambios propios mediados por la tecnología, por consiguiente, se debe estar al tanto de los requerimientos tecnológicos en el quehacer pedagógico, pensando continuamente en el cambio de sus estrategias didácticas en el proceso de enseñanza con el uso adecuado de los recursos tecnológicos. En este aspecto es importante tomar como referente los estándares de competencias TIC para docentes de la UNESCO, el documento de Competencias TIC para el desarrollo profesional docente del MEN de Colombia, y algunos estudios sobre formación en competencias TIC para profesores de básica primaria.

Respecto a las competencias TIC es fundamental referenciar a la UNESCO (2008) con su documento Estándares de Competencias en TIC para docentes, cuya finalidad pretende:

Mejorar la práctica de los docentes en todas las áreas de su desempeño profesional, combinando las competencias en TIC con innovaciones en la pedagogía, el plan de estudios (currículo) y la organización escolar; aunado al propósito de lograr que los docentes utilicen competencias en TIC y recursos para mejorar sus 
estrategias de enseñanza, cooperar con sus colegas y, en última instancia, poder convertirse en líderes de la innovación dentro de sus respectivas instituciones. (p.4.)

De igual manera, el documento de estándares de competencias en TIC propuesto por la UNESCO propone tres enfoques importantes a saber: Nociones básicas de TIC, Profundización del conocimiento y generación del conocimiento. Cada enfoque se soporta en cinco componentes del sistema educativo: pedagogía; práctica y formación profesional de docentes; plan de estudios (currículo) y evaluación; organización y administración de la institución educativa; y uso de las TIC. Esto dirigido a los profesores de básica primaria, básica secundaria, media técnica y todos los actores vinculados en la educación, con el fin de mejorar la calidad educativa.

Para el caso de Colombia el Documento "Competencias TIC para el desarrollo profesional docente" publicado en el año 2013 por el MEN pretende "guiar el proceso de desarrollo profesional docente para la innovación educativa pertinente con uso de TIC; están dirigidas tanto para quienes diseñan e implementan los programas de formación como para los docentes y directivos docentes en ejercicio" (p.29), y está fundamentado en cinco principios: pertinente, práctico, situado, colaborativo e inspirador. También cuenta con tres niveles: exploración, integración e innovación, y cinco competencias: tecnológica, pedagógica, comunicativa, de gestión e investigativa.

Ante la preocupación por la integración de las TIC en los procesos de enseñanza y aprendizaje, el Ministerio de las Tecnologías de la Información y las Comunicaciones, el Ministerio de Educación Nacional y Computadores para Educar, han brindado a los profesores estrategias pedagógicas y de gestión innovadoras mediadas por las TIC, que les permitan acceder a nuevos conocimientos, experiencias de aula y contenidos educativos digitales, esto con el fin de fortalecer y transformar sus prácticas pedagógicas integrando las TIC. En abril de 2020 el Ministerio TIC, Computadores para Educar, el MEN, y otros aliados, abrieron la convocatoria para profesores, con el fin de capacitarlos en pensamiento crítico y computacional y ampliar así sus conocimientos frente a las nuevas tecnologías. También con el propósito de estimular las habilidades de los profesores, para que ellos desde sus prácticas pedagógicas puedan innovar y desarrollar competencias de pensamiento crítico, competencias digitales y desarrollo de la creatividad en sus estudiantes.

Respecto a la formación en competencias TIC para profesores de básica primaria, es importante resaltar que la mayoría de profesores que orientan la educación primaria son licenciados en diferentes áreas del conocimiento y no todos están cualificados para integrar pedagógicamente las TIC, lo que requiere compromiso y autoformación por parte de cada uno de ellos, ya que integrar las TIC a la práctica pedagógica requiere un cambio en estrategias didácticas y metodológicas, por lo que Trigueros, Sánchez y Vera (2012) consideran que es importante:

Conocer si el profesor las utiliza como apoyo a su tarea docente, como recurso y como medio para conseguir los objetivos propuestos o, al contrario, sólo se ciñe al libro y a otros elementos más cercanos para él, más fáciles de usar y que no requieren los cambios metodológicos que requieren el uso de las TIC. (p. 102)

Pese a que en la actualidad se habla de cultura digital, competencias TIC, y formación de profesores en TIC, es importante resaltar la percepción de Prensky (2010) cuando manifiesta la preocupación por las nuevas generaciones que tienen la "sensación de que a las aulas han llegado, para instruirles, un nutrido contingente de extranjeros que hablan idiomas desconocidos, extranjeros con muy buena voluntad, sí, pero ininteligibles" (p.6). Por esta razón, los profesores como protagonistas en el proceso de enseñanza deben estar formados y actualizados para aplicar estas tecnologías en sus prácticas pedagógicas.

De la misma forma, es fundamental reconocer que los profesores que orientan básica primaria en muchas instituciones trabajan con aula multigrado y por consiguiente "deben atender de manera simultánea en un aula estudiantes del nivel de preescolar y de los cinco grados de la básica primaria, orientando las diez áreas propuestas por el sistema educativo colombiano" (Uribe, 2015, p.19). En este sentido, la integración de las TIC se presenta como una oportunidad de dinamizar sus prácticas e integrar a los estudiantes para que sean ellos quienes las apropien y puedan desarrollar aprendizaje autónomo. 


\section{Metodología}

El enfoque que privilegió el desarrollo de esta investigación fue cualitativo, ya que permite dar cuenta de la comprensión de fenómenos educativos y sociales y es "fenomenológica porque enfatiza los aspectos subjetivos del comportamiento humano, el mundo del sujeto, sus experiencias cotidianas, sus interacciones sociales y los significados que da a esas experiencias e interacciones" (Moreira, 2002. p.5). Se retoma la Investigación Acción Pedagógica, dado que este tipo de investigación facilita la elaboración del saber pedagógico, donde los profesores evidencian su práctica diaria, de manera que responda en forma adecuada a las condiciones del medio, a las necesidades de aprendizaje de los estudiantes y al entorno sociocultural de cada uno de ellos. Para Restrepo (2004), la Investigacion Acción Pedagógica :

Está más ajustada al quehacer de cada profesor y se va construyendo mediante la reflexión acerca de la práctica en la acción de todos los días y en transformación permanente de aquella y su relación con los componentes disciplinares que la determinan (p.47).

La investigación se desarrolló con un grupo de siete (7) profesores de primaria pertenecientes a la Institución Educativa Técnica Agrícola (IETA) de Paipa. La totalidad de los participantes son licenciados en básica primaria y han desarrollado estudios de posgrado. El $47 \%$ cuenta con una experiencia laboral de más de veinte años y el 57 $\%$ una experiencia entre 10 y 20 años.

\subsection{Contexto}

La IETA se encuentra ubicada en el perímetro rural del municipio de Paipa, Boyacá, en la vereda del Salitre. Sus siete (7) sedes se encuentran ubicadas en las veredas de Alto Plateado, Quebrada Honda, La Playa, La Esperanza, San José, El Tunal e Isidro Rivera. Sus estudiantes reciben una formación con orientación agropecuaria y modelo pedagógico constructivista, enfoque pedagógico de aprendizaje significativo combinado con escuela nueva; la institución tiene articulación con el Servicio Nacional de Aprendizaje (SENA) regional Boyacá CEDEAGRODuitama, por lo tanto, sus graduandos reciben el título de Bachiller Técnico con Especialidad Agropecuaria (Institución Educativa Técnica Agrícola de Paipa, 2018).

Figura 1

Ubicación de la IETA en el municipio de Paipa.

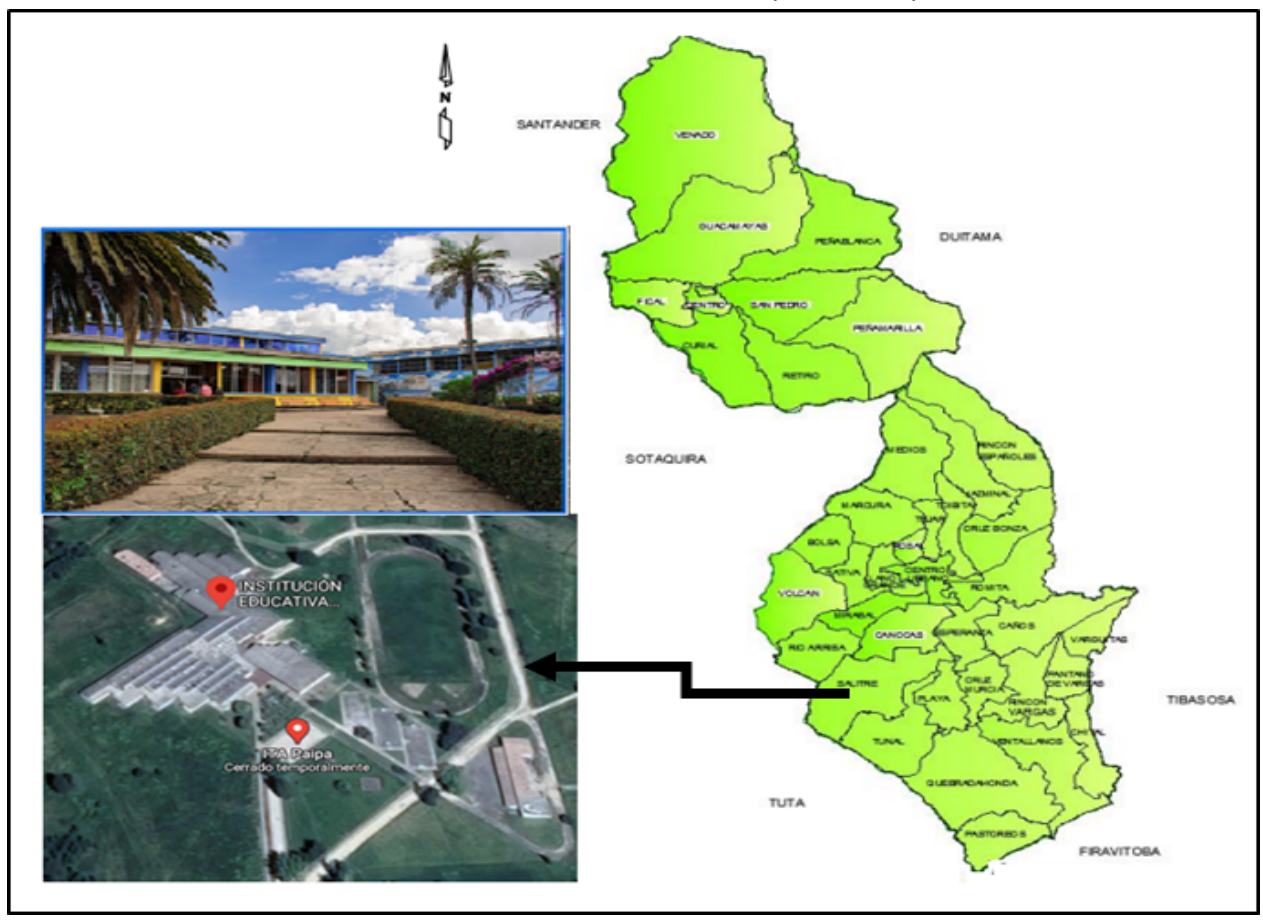

Fuente: Google Maps (2020) y Gobernación de Boyacá (2020) 


\subsection{Momentos de la investigación}

La investigación se desarrolla en tres momentos: primero, la reflexión de la práctica pedagógica con los profesores de la IETA, reflexión que retoma dos aspectos: la contextualización de la institución educativa y el análisis de la práctica fundamentada en cuatro (4) categorías: los propósitos, contenidos, estrategias metodológicas y la evaluación. Un segundo momento, referente a la propuesta colectiva de acciones pedagógicas renovadoras con los profesores de la IETA, donde se estructura el programa de formación y, un tercer momento correspondiente a la valoración del programa respecto a competencias TIC.

Figura 1

Momentos de la Investigación

MOMENTOS DE LA METODOLOGía
Deconstrucción de las prácticas
pedagógicas de los profesores de la
Institución Educativa Técnica Agrícola
mediadas por las TIC.
Reconstrucción de las prácticas
pedagógicas
Evaluación de las transformaciones
pedagógicas sobre formación de
profesores en competencias TIC para la
enseñanza de la estadística

ACTIVIDADES

Categorización de las prácticas pedagógicas a través de un estado del arte. Cuestionario estructurado a estudiantes y profesores, Diagnóstico sobre competencias TIC.

Análisis colectivo sobre los resultados obtenidos en el momento de la deconstrucción.

Valoración de la práctica pedagógica implementada, a través de satisfacción personal de profesores frente a los cambios observados en los lanes de área y Diarios de campo

Fuente. Elaboración propia

\section{Resultados}

\subsection{Repensando la práctica pedagógica con los profesores}

Para reflexionar la práctica pedagógica con los profesores de la IETA, se tomaron como referente los componentes para la formación de profesores propuestos por Quero (2006), que hacen referencia al análisis desde la práctica y el saber pedagógicos, y donde se tienen en cuenta cuatro (4) componentes: el currículo, los docentes, los alumnos y el proceso formativo. A partir de lo dicho por Quero, en el proceso de investigación con los profesores de la IETA se analizaron los planes de área teniendo en cuenta las preguntas orientadoras de Coll (2006, citado por De Zubiria) ¿Para qué enseñar?, ¿qué enseñar?, ¿cómo enseñar? y ¿qué, cómo y cuándo evaluar?; basado en lo dicho por Quero y Zubiría se identificaron las categorías que fundamentan la investigación sobre las cuales se reflexionó la práctica pedagógica con los profesores de la IETA. Estas categorías corresponden a: propósitos, contenidos, y estrategias de enseñanza y evaluación, como se observan en la siguiente figura. 
Figura 2

Categorías sobre las cuales se analizaron las

prácticas pedagógicas de los profesores de la IETA
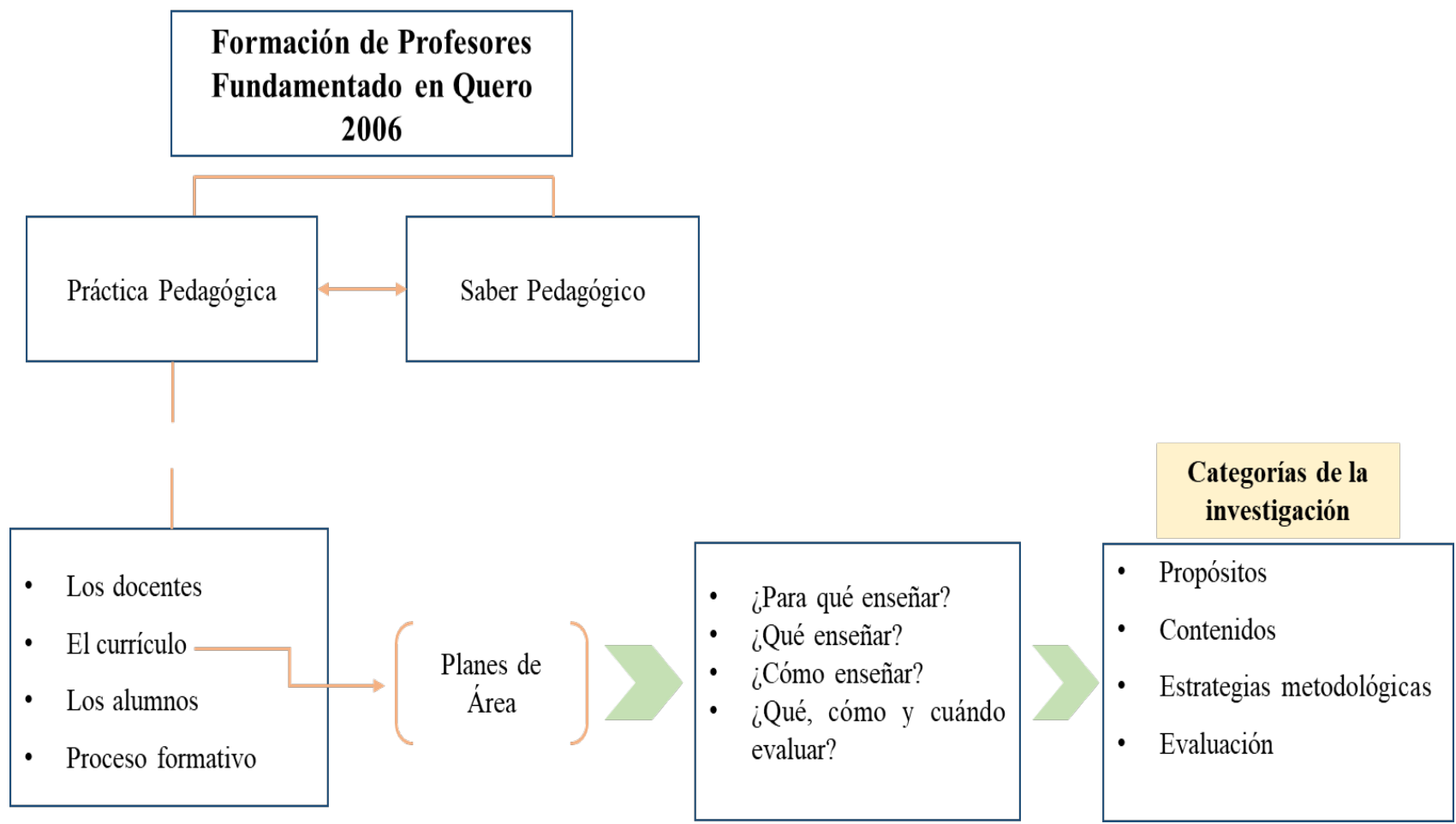

Fuente: Elaboración propia a partir de Quero (2006) y Coll (2006)

Habiendo definido las categorías, se hizo un análisis sobre el currículo como un componente importante en la práctica pedagógica. Quero (2006) menciona:

En las instituciones educativas existen tres versiones (a) un currículo oficial, prescrito por las autoridades educativas, (b) un currículo oculto, derivado de las rutinas, prácticas y costumbres que se dan en la institución y (c) un currículo real como expresión del balance de los dos anteriores. (p.91)

En el contexto de la IETA, el currículo oficial está dado por el plan de estudios que se realiza al comienzo del año lectivo, teniendo en cuenta los lineamientos del MEN, y que se va reconstruyendo en el transcurso de este. El currículo oculto está supeditado a las estrategias metodológicas de cada profesor y a las necesidades de la comunidad educativa; y el currículo real es el trabajo que los profesores desarrollan a diario en el aula, ya que con frecuencia ocurre que el profesor planea sus actividades y termina desarrollándolas de manera diferente de acuerdo con los intereses de sus estudiantes "lo que indica que es una cosa la que nos dice el programa que enseñamos; otra la que realmente enseñamos y otra distinta la que aprenden los alumnos" (Quero, 2006. p.91).

Referente a los alumnos, los profesores en sus prácticas pedagógicas deben reflexionar sobre aspectos como: ¿Los tratamos con respeto?, ¿consideramos sus opiniones?, ¿leemos con interés sus trabajos?, ¿los involucramos en el proceso de enseñanza y aprendizaje?, entre otros. Con respecto al aprendizaje de los profesores, se puede ver desde la formación académica recibida por las universidades, la formación que se da en el ejercicio de la profesión docente y la formación personal de los profesores con ciertos vacíos en dicha formación.

Retomando lo expuesto respecto a la reflexión de las practicas pedagógicas se tomó la primera categoría referente a los Propósitos donde se reflexionó aspectos institucionales como el Proyecto Educativo Institucional (PEI), evidenciando que la implementación de las TIC no está articulada en su totalidad en el PEI y en los planes de área solo se enfatiza en el área de tecnología e informática. Referente al perfil del estudiante, los profesores 
orientan a sus educandos para identificar las características predominantes de su dimensión personal, así como su estilo de aprendizaje, y de esta manera reflexionar con ellos sobre sus fortalezas y áreas de oportunidad.

En cuanto al trabajo en equipo, los resultados de esta etapa permitieron evidenciar que las interacciones que se construyen a partir de las relaciones entre profesores, se observarán para verificar el favorecimiento de los procesos de enseñanza, el ambiente que se genera en relación al quehacer pedagógico dentro del empleo de los diferentes espacios que se tienen para beneficiar dichos procesos, la construcción colectiva del aprendizaje, vivencias, reflexiones, experiencias, convivencia, comunicación, y sobre todo relaciones significativas, cotidianas y reciprocidad entre profesores. El resultado fue provechoso al encontrar que existe empatía entre las partes y motivación por un diseño de estrategias pedagógicas adecuadas para fortalecer el trabajo en equipo.

En lo referente al clima escolar se evidenció que los profesores participantes en la investigación hacen acuerdos (pactos de aula) al inicio de cada periodo académico para generar una buena convivencia en la comunidad educativa, todo esto con el fin de reafirmar valores como el respeto, la tolerancia, la aceptación de diferencias, el derecho a la opinión sin temor a sentirse burlados por los demás y el trabajo en equipo, entre otros. En cuanto al ambiente para el aprendizaje, los salones se encuentran distribuidos en rincones del saber dotados de buenos mobiliarios, y los recursos usados para la enseñanza se encuentran bien ubicados.

Como segunda categoría sobre los contenidos, se resalta su importancia teniendo en cuenta que hacen parte de los planes de área contemplados en el currículo, ya que promueven el aprendizaje significativo haciendo parte del proceso cognitivo. De igual manera, desde la práctica pedagógica los contenidos debe ser una buena base para la innovación de la enseñanza. Cabero y Marín (2014) afirman que "los profesores deben conocer los contenidos que deben enseñar, los hechos, los conceptos y sus teorías" (p. 19). Es importante resaltar que en la reflexión colectiva respecto a los contenidos, los docentes manifiestan la necesidad de recibir formación en competencias TIC para dinamizar las prácticas pedagógicas en la enseñanza de la estadística, teniendo en cuenta que esta materia es un componente fundamental de las matemáticas y todos los profesores participantes la orientan en sus aulas.

De igual manera, es importante recalcar que las TIC han generado un gran impacto en el campo educativo, donde los profesores de esta época enfrentan cambios propios mediados por la tecnología, por consiguiente, se debe estar al tanto en los requerimientos tecnológicos en el quehacer pedagógico, pensando continuamente en el cambio de estrategias didácticas en el proceso de enseñanza con el uso adecuado de los recursos tecnológicos.

En cuanto a la estadística como rama de las matemáticas, es importante desarrollar procesos de formación para los profesores, de tal forma que apropien las competencias necesarias para la incorporación de las TIC en la enseñanza, donde el profesor sea un mediador y el estudiante sea el protagonista de su propio aprendizaje. Castillo (2008) manifiesta que "las TIC pueden apoyar a los estudiantes en varios ejes matemáticos, como números, medida, geometría, estadística, álgebra, pues se espera que cuando dispongan de ellas logren concentrarse en tomar decisiones, razonar y resolver problemas" (p.185).

Referente a la formación de profesores en estadística implementando las TIC, se requiere de un proceso de formación, porque la gran mayoría de docentes de primaria no son licenciados en matemáticas y tampoco fueron formados en aplicaciones TIC para la implementación de la estadística. "un punto esencial para introducir la tecnología en la clase de estadística, la adecuada preparación de los profesores, tanto desde el punto de vista técnico como didáctico (Batanero, Arteaga, Contreras, 2011, p.15)

En lo que refiere a los contenidos, se pudo evidenciar que los profesores de la IETA tienen claridad en los mismos referentes al área de matemáticas, ya que se basan en los estándares de competencias y los Derechos Básicos para el Aprendizaje- DBA- estipulados por el MEN; la dificultad que manifestaron los docentes está enfocada en la apropiación de las TIC para dinamizar los procesos de enseñanza en la estadística. Los profesores también 
expresan que adaptan los contenidos a los ritmos de aprendizaje de los estudiantes, desarrollando actividades dentro y fuera del salón de clase.

Como tercera categoría están las estrategias metodológicas, las cuales se contemplan en el plan de área y están relacionados con las actividades que los profesores crean y desarrollan en sus prácticas de enseñanza. Respecto al modelo pedagógico, se encontró que los profesores tienen en cuenta el modelo pedagógico constructivista de la institución, de manera que logran apoyar al estudiante para fortalecer el trabajo colaborativo, ser capaces de auto dirigirse, autoevaluarse y auto monitorearse, tener habilidades de autoaprendizaje que les permitan aprender para la vida, saber resolver problemas, ser empático, flexible, creativo y responsable.

En cuanto a las normas de convivencia, los profesores inculcan comportamientos, actividades y saberes en condiciones lógicas expresadas en sus prácticas pedagógicas, sin necesidad de ceñirse a normas, reglas o códigos, y a través de sus experiencias, siendo un producto del trabajo pedagógico esta enseñanza que se realiza en toda acción pedagógica, lo cual crea en los estudiantes hábitos de comportamiento que se verán reflejados en su toma de decisiones y razonamientos lógicos.

En el cuestionario aplicado a los profesores sobre prácticas pedagógicas y TIC, en lo concerniente al uso de recursos tecnológicos en el aula para el desarrollo del área de estadística, los resultados obtenidos demostraron que los profesores de la IETA cumplen con los requerimientos de las prácticas pedagógicas, ya que los valores obtenidos evidencian su aplicación y apropiación en este sentido; no obstante, se recomienda que los profesores incrementen el uso de los recursos tecnológicos, con el fin de hacer más fácil y didáctico el proceso de enseñanza.

En cuanto al uso de recursos tecnológicos en las clases, los resultados obtenidos mostraron que existe poca apropiación del uso de los diferentes recursos tecnológicos, lo que deja ver que en este sentido existe un mediano aprovechamiento de estos. De ahí que el acto comunicativo inmerso en el proceso pedagógico bajo el ambiente de las TIC se esté viendo mermado, por tal motivo se requiere del compromiso personal e institucional en el desarrollo de habilidades para el manejo de herramientas TIC, lo que si hace con sentido de pertenencia y responsabilidad logra que la formación profesional integral propenda por un mejor ambiente de aprendizaje al interior del aula de clase.

Respecto a las competencias TIC, teniendo en cuenta los resultados obtenidos en el taller diagnóstico aplicado a los profesores, el cual corresponde al instrumento que se desarrolló para evaluar las competencias TIC (basado en la guía No. 1 de los anexos de los estándares de competencias TIC para el desarrollo profesional docente del MEN), se puede evidenciar que los docentes no poseen conocimientos básicos sobre las competencias tecnológicas, encontrándose en el nivel explorador, es decir, que reconocen una serie de herramientas tecnológicas pero no las integran en sus prácticas pedagógicas.

La cuarta y última categoría la evaluación, hace referencia a un proceso que permite identificar los avances en la construcción de las competencias fundamentales en cada uno de los niveles y grados adoptados institucionalmente, a partir de las etapas del desarrollo de los y las estudiantes y del plan de estudios, específicamente a través del alcance de los desempeños. Es un proceso permanente por medio del cual se busca monitorear, valorar y emitir juicios sobre el desarrollo del o de la estudiante y el progreso de sus habilidades y competencias de acuerdo con los estándares PEI-IETA.

Los profesores tienen en cuenta las características de la evaluación en el momento de valorar el aprendizaje de los estudiantes, tomando como referente lo estipulado en el Sistema Integral de Evaluación y Promoción de Estudiantes - SIEPE de la IETA. En este se enfatiza sobre la evaluación de forma integral teniendo en cuenta los componentes del aprendizaje: lo cognitivo, procedimental y actitudinal. La evaluación está centrada en el ritmo de aprendizaje de los estudiantes sin descuidar la calidad; la evaluación es continua y permanente, y los profesores hacen acompañamiento y seguimiento de las fortalezas y debilidades durante dicho proceso. 
Los profesores manifiestan proporcionar a los estudiantes una clara orientación acerca de la evaluación, lo que permite identificar los avances en la construcción de habilidades y competencias y valorar el desempeño de los estudiantes en su proceso de aprendizaje, realizar la respectiva realimentación y establecer estrategias de nivelación con el fin de mantener la calidad de la formación integral. Así mismo, los profesores preparan guías con orientaciones claras sobre la forma de evaluar y hacer la respectiva realimentación para ajustar sus metodologías a las necesidades de la comunidad educativa.

Al concluir el análisis de las prácticas pedagógicas con los profesores de la IETA, se pudo establecer que los profesores presentan fortalezas respecto al comportamiento social de sus estudiantes, haciendo énfasis en los valores, buenos modales, respeto por las diferencias, inclusión, entre otros, lo que contribuye a una sana convivencia y a un adecuado clima escolar. Dentro de los aspectos por mejorar se pudo observar que no se hace uso de los recursos tecnológicos en sus prácticas pedagógicas y las TIC no están integradas en los planes de área.

\subsection{Propuesta colectiva de prácticas pedagógicas renovadoras}

Referente a las acciones pedagógicas renovadoras, se planteó una reconstrucción de la práctica pedagógica con los profesores de la IETA y en conjunto se tomó la decisión de fortalecer el proceso de formación en las competencias TIC para la enseñanza de la estadística, teniendo presente lo expuesto por los profesores en la reflexión de la práctica pedagógica, donde manifestaron la necesidad de conocer, interactuar e integrar herramientas TIC en la enseñanza de la estadística, ya que se hace necesario fortalecer competencias digitales en los profesores.

En cuanto al recurso TIC para dinamizar la enseñanza de la estadística, se acordó con los profesores trabajar Excel, ya que es "un recurso didáctico potente y muy útil, que nos permite conseguir una aproximación más exploratoria y significativa en la enseñanza de la estadística" (Noriega y Huerta 2006, p.5), en esta medida Excel permite desarrollar diferentes actividades acordes a la temática, además de ser un programa que viene en el paquete de Office, por tal razón todos los computadores permiten su fácil acceso. Igualmente presenta una interfaz gráfica amigable e intuitiva que facilita el desarrollo de diferentes actividades desde distintas áreas del conocimiento. Cabe resaltar que en el proceso de formación además de Excel, también se hizo orientación del editor de texto Word, editor de presentaciones PowerPoint y Compucol "plataforma de notas institucional", a solicitud de los profesores participantes.

EL programa de formación de profesores, se realizó en dieciséis sesiones, durante el desarrollo de cada una de ellas se determinó una temática, acompañada de una serie de actividades, entre las que se incluyeron: presentación de la propuesta, cuestionarios tanto para profesores como para estudiantes, taller diagnóstico a profesores para conocer el nivel de competencia que poseen al momento de aplicar tecnologías de la información, conversatorios para determinar las fortalezas y las debilidades, aplicación de la herramienta Excel, Word, PowerPoint, la plataforma Compucol y realimentación para evaluar la propuesta planteada. Estas sesiones se llevaron a cabo en la sala de informática de la IETA, en cafés de internet del municipio de Paipa, en la Biblioteca Municipal y en algunas de las sedes de la institución. Para el desarrollo del proceso de formación, se diseñó un cronograma de actividades por sesiones, se llevó registro en diarios de campo sobre los avances alcanzados por los profesores en cada sesión, de tal forma que esas apreciaciones facilitaran emprender el curso de forma significativa.

De igual manera, en el proceso de formación se evidenció que los profesores mostraron avances respecto a las habilidades en las competencias TIC, que inicialmente se acordó abordar en la formación. En cuanto a la competencia tecnológica se pudo ver la disposición de los profesores por el uso e implementación de las TIC; en la pedagógica se exaltó la experiencia de los profesores, el dominio del área, el manejo curricular, entre otros y; en la competencia comunicativa se observó la significatividad y el sentido práctico de lo aprendido. Así mismo, 
se evidenció que los profesores de básica primaria de la IETA integraron en su plan de área actividades mediadas por las TIC para desarrollar procesos de enseñanza de la estadística con sus estudiantes.

Finalmente, se evidenció que los profesores de básica primaria de la IETA presentan disposición para trabajar en grupo, carisma, deseo de servicio y colaboración; haciendo de este proceso un tiempo de trabajo y crecimiento permanente. Cabe resaltar que dentro de los valores que más se destacan está el respeto, el cual se evidenció en cada encuentro al exponer sus ideas, hacer sugerencias o al recibir información. Del mismo modo, el compañerismo fue otro aspecto relevante, por cuanto el apoyo manifiesto en las actividades fue mutuo, quienes tenían mayores habilidades orientaron a los compañeros que presentaban alguna dificultad, situación que generó confianza y seguridad entre el grupo, logrando un clima agradable para el desarrollo de las distintas actividades.

\subsection{Valoración de la práctica pedagógica renovadora respecto a competencias TIC}

La valoración de las prácticas pedagógicas renovadoras con los profesores de básica primaria de la IETA fue vista a partir de la experiencia en el proceso de formación con los docentes y los datos registrados en los diarios de campo en cada una de las sesiones.

A partir del análisis de los resultados, se identificó el nivel de transformación pedagógica que obtuvieron los profesores de la IETA. Se observó que un alto porcentaje de los profesores tienen más elementos de juicio para encaminar sus labores pedagógicas, se realizaron ajustes en los planes de área donde se evidenció el uso de las TIC, la planeación de clases presenta actividades que facilitan a los estudiantes la integración de las TIC para su desarrollo, fortaleciendo en ellos el análisis crítico, analítico y propositivo. De esta manera, se formarán estudiantes proactivos que propendan por un futuro mejor, no sólo para ellos sino para las comunidades donde lleguen a interactuar. En cuanto a la relación que se debe dar entre lo enseñado y lo aprendido, tal como sucede con la motivación a realizar debates críticos, diálogos o interpelaciones, se muestra que en estos aspectos tuvieron una aceptable transformación pedagógica, por lo que se hace necesario trabajar en este aspecto un poco más.

La organización de ambientes según las actividades propuestas fue otro factor cuyo resultado fue importante, ya que los profesores manifiestan que el proceso de formación aportó ideas significativas para la estructuración de estos. La gran mayoría de profesores participantes en la investigación trabajan en aulas o salones con espacios reducidos y demasiado cerrados, esto hace que si el ambiente no se organiza de manera adecuada, en lugar de captar la atención termina por distraer a los estudiantes, de ahí que los profesores en su experticia son quienes determinan la ubicación de cada elemento así como de cada estudiante.

Por ende, además de tener dos o hasta tres cursos, hay que tener en cuenta las edades y las necesidades de aprendizaje de cada estudiante, así como la distribución del material y por supuesto la orientación académica acorde con los grados en que se orienta. Del mismo modo, la decoración del aula juega a favor, ya que si bien es cierto las imágenes pueden distraer, también es bien sabido que un buen panorama visual estimula la creación, la productividad, la atención y hasta dispersa la agresividad.

Así mismo, los rincones del saber son de suma importancia ya que el material académico que allí se dispone, permite que el estudiante en su autonomía dirigida acceda a la información y al conocimiento de manera más agradable. Es allí donde en compañía de los demás compañeros, se conjugan otros elementos como el trabajo en equipo, la toma de decisiones, la resolución de inquietudes y de conflictos, factores todos ellos que hacen que la vida en comunidad de los estudiantes se torne en uno de los fundamentos más significativos del ambiente escolar. 
Igualmente, se vio reflejado el uso de herramientas TIC aplicadas en el aula y la motivación que estas generan en los estudiantes, demostrando su excelente transformación pedagógica. Es de resaltar la disposición de los profesores para seguir aprendiendo, el deseo de superación por enriquecer el dominio de las herramientas TIC es notorio y se refleja en las actividades ejecutadas. Se enfatiza en la aplicación de herramientas al momento de hacer exposiciones, elementos que sin lugar a duda son de gran ayuda por cuanto facilitan la labor del docente. Cabe resaltar que el aspecto tecnológico si bien requiere de máxima atención y práctica, fue realizado de manera muy adecuada por parte de los profesores.

Otra fortaleza evidenciada fue el aspecto comunicativo en el que los docentes no solo optimizaron la comunicación asertiva en la convivencia escolar sino también en el proceso de enseñanza con el uso de herramientas tecnológicas. En este caso con la implementación del programa Excel se fortaleció la capacidad de interpretar, describir, clasificar y representar datos estadísticos; al igual con el uso de plataformas y la creación de ilustraciones que se convirtieron en fuentes de intercambio de información.

\subsection{Aspectos orientadores para la formación de profesores de primaria en competencias TIC}

Una vez desarrollada esta investigación y como producto de la experiencia vivida con los profesores participantes, aunado a lo dicho por varios autores referenciados en este proyecto, se logró identificar algunos aspectos que deben tenerse en cuenta para un programa de formación de profesores que busque dinamizar las prácticas pedagógicas en básica primaria:

- Caracterización del contexto institucional: familiarizarse con el contexto en el cual se encuentra la población objeto, permite enfocar de una u otra manera los procesos de formación, ya que no es lo mismo orientar un proceso de formación para profesorado de contexto rural que para profesorado de contexto urbano. Por ende es fundamental conocer el contexto institucional y regional, apoyándose en la experiencia de los profesores para que la formación logre el objetivo propuesto.

- Caracterización de los profesores: conocer al profesorado con el cual se va a trabajar es de gran importancia, ya que a partir de esta caracterización se conocen detalles referentes a su formación académica, tiempo de experiencia, asignaturas que orienta, entre otros aspectos que facilitan enfocar acciones colectivas para reflexionar y reconstruir las prácticas pedagógicas.

- Diagnóstico sobre competencias TIC: conocer el nivel de competencias TIC en el cual se encuentran los profesores es fundamental, ya que a partir de este diagnóstico se tiene un referente para proponer un programa de formación que sea significativo para todos los profesores participantes, aprovechando las fortalezas de cada uno de ellos y potencializando competencias para que puedan integrar las TIC en sus prácticas de aula, facilitando la dinamización de los procesos de enseñanza y aprendizaje.

- Reflexión de profesores sobre sus prácticas pedagógicas: es importante que los profesores reflexionen de forma individual y colectiva sobre la actividad diaria que desarrollan en el aula, ya que este espacio de reflexión permite un cambio de estrategias metodológicas donde las TIC son de gran importancia. Así mismo se deben tener en cuenta las necesidades de los estudiantes y el dominio curricular, que es fundamental para reflexionar sobre ¿Qué se enseña y cómo se enseña?, porque a partir de esta reflexión los profesores identifican las dificultades y generan trasformación en sus prácticas en el aula.

- Actualización continua de planes de área: la revisión y actualización de los planes de área, como proceso de formación de profesores en competencias TIC para básica primaria, es un referente inicial para identificar cómo los profesores están integrando las TIC en su planeación. Esto a su vez posibilita la reflexión individual y colectiva de las prácticas contribuyendo a identificar y analizar las categorías sobre las cuales se pueden reconstruir y valorar las prácticas pedagógicas.

1. Uso significativo de recursos tecnológicos: entender y utilizar pedagógicamente los recursos tecnológicos permite la dinamización y motivación de los procesos de aprendizaje e incentiva en los estudiantes el trabajo 
autónomo y el desarrollo de la creatividad. Por tal razón los profesores deben tener las competencias que permitan dar uso significativo a estos recursos en sus prácticas pedagógicas.

2. Fortalecer el trabajo en equipo: trabajar en equipo es un esfuerzo de todos los miembros del grupo para alcanzar objetivos comunes, y en la educación como en muchas otras áreas, trabajar en equipo es fundamental ya que permite que los profesores interactúen fácilmente con sus colegas y a la vez promuevan comunidades de aprendizaje que contribuyan a fortalecer el conocimiento; factores que incentivan la capacidad para renovar e innovar en las prácticas pedagógicas con el fin de mejorar en los procesos de enseñanza y aprendizaje tanto de estudiantes como de profesores.

\section{Conclusiones}

La integración de las TIC en las prácticas pedagógicas permite cambios en las estrategias metodológicas, generan procesos de innovación desde todas las áreas y fortalece el quehacer pedagógico de los profesores; de igual manera, promueve en los estudiantes la creatividad, el trabajo autónomo y la toma de decisiones.

Reflexionar las prácticas pedagógicas mediadas por las TIC con los profesores de la IETA, fue un factor importante para la transformación e innovación de los procesos de enseñanza y aprendizaje, se logró integrar las tecnologías de forma pedagógica y enriquecer el saber pedagógico de los profesores, quienes a partir de este saber reflexionaron y plantearon estrategias metodológicas que facilitan a sus estudiantes competencias para construir su propio conocimiento.

La metodología de Investigación Acción Pedagógica posibilita hacer una reflexión colectiva de las prácticas pedagógicas desde y con los profesores, permitiendo evidenciar fortalezas y aspectos por mejorar, sobre los cuales se puede luego hacer una reestructuración de las prácticas que conlleve a la trasformación de estas y finalmente una valoración para evidenciar y evaluar la efectividad del proceso de formación de profesores.

El programa de formación con los profesores permitió identificar aspectos importantes que se deben tener en cuenta para abordar procesos significativos de formación en TIC, estos aspectos hacen referencia a comprender el contexto institucional, conocer a los profesores, saber en qué nivel de competencias TIC se encuentran, reflexionar colectivamente la práctica pedagógica, hacer ajustes en los planes de área y fortalecer el trabajo en equipo, de esta manera se logran establecer comunidades de aprendizaje donde se compartan experiencias metodológicas y didácticas que ayuden a la autoformación e innovación en los procesos de enseñanza y aprendizaje.

La significación de un proceso de formación se fortalece con el apoyo de los directivos docentes y de los profesores participantes que sean abiertos y propositivos frente a los programas innovadores. En el desarrollo de este proyecto se evidenció como los profesores mejoraron el trabajo en equipo, el intercambio de saberes, y por ende sus metodologías en los procesos de enseñanza y aprendizaje implementando las TIC.

La formación de profesores en TIC es fundamental para ofrecer una educación de calidad, teniendo en cuenta no sólo la situación vivida en la actualidad por causa de la pandemia, sino porque el mundo está inmerso en las tecnologías, donde niños y jóvenes cada vez se sienten más atraídos por ellas, por lo que no se puede desconocer las bondades que éstas brindan no sólo como herramientas de entretenimiento, sino como herramientas de comunicación y aprendizaje.

\section{Referencias bibliográficas}

Batanero , C., Arteaga, P., y Contreras, M. (2011). El currículo de estadística en la enseñanza obligatoria. EM TEIA - Revista de Educação Matemática e Tecnológica Iberoamericana, 2(2), 1-20. Obtenido de http://www.ugr.es/ jmcontreras/pages/Investigacion/articulos/2011EmTEia.pdf 
Cabero, J. (2010). Los retos de la integración de las TICs en los procesos educativos. Límites y posibilidades. Perspectiva Educacional. Foamción de profesores, 49, 32-61. Obtenido de http://www.perspectivaeducacional.cl/index.php/peducacional/article/view/3

Cabero, J., y Marín, V. (2014). Miradas sobre la formación del profesorado en tecnologías de información y comunicación (TIC). Enl@ce: Revista Venezolana de Información de Tecnología y Conocimiento, 11(2), 1124. Obtenido de https://www.redalyc.org/articulo.oa?id=82332625005

Castillo, S. (2008). Propuesta pedagógica basada en el constructivismo para el uso óptimo de las TIC en la enseñanza y el aprendizaje de la matemática. Revista latinoamericana de investigación en matemática educativa, 11(2), 171-194. Obtenido de http://www.scielo.org.mx/pdf/relime/v11n2/v11n2a2.pdf

Danielson, C. (2013). Enhancing Professional Practice: A Framework for Teaching (2 ed.).

Davini, M. (2008). Acerca de las prácticas docentes y su formación. Dirección Nacional de Formación e Investigación. Buenos Aires: Ministerio de Educación de Argentina. Obtenido de http://www.bnm.me.gov.ar/giga1/documentos/EL005899.pdf

De Zubiria, J. (2006). Los modelos pedagógicos. Hacia una pedagogía dialogante. Magisterio.

Díaz, V. (2006). Formación docente, práctica pedagógica y saber pedagógico. Laurus Revista de Educación, 12(Extraordinario), 88-103. Obtenido de https://www.redalyc.org/pdf/761/76109906.pdf

Domingo, M., y Marquès, P. (2011). Aulas 2.0 y uso de las TIC en la práctica docente. Revista Científica de comunicación y educación Comunicar, XIX(37), 169-175. doi:https://doi.org/10.3916/C37-2011-03-09

López, M., Lagunes, C., y Herrera, S. (2006). Excel como una herramienta asequible en la enseñanza de la Estadística. Teoría de la Educación. Teoría de la Educación. Educación y Cultura en la Sociedad de la Información, 7(1), 1-9. Obtenido de https://www.redalyc.org/pdf/2010/201021084007.pdf

Marcelo, C. (1995). Formación del profesorado para el cambio educativo. Barcelona: Promoción y Publicaciones Universitarias.

Ministerio de Educación Nacional. (2013). Competencias TIC para el Desarrollo profesional Docente (Primera ed.). Bogotá: Imprenta Nacional. Obtenido de http://www.premiosantillana.com.co/pdf/competencias_tic.pdf

Ministerio de Educación Nacional. (12 de Diciembre de 2019). Ministerio de Educación Nacional. Obtenido de https://www.mineducacion.gov.co/1759/articles-357388_recurso_1.pdf

Moreira, M. A. (2002). Investigación en educación en ciencias: métodos cualitativos. Actas de PIDEC (págs. 129). Porto Alegre: Universidade Federal do Rio Grande do Sul. Obtenido de http://www.if.ufrgs.br/ moreira/metodoscualitativos.pdf

Prensky, M. (2010). Nativos e Inmigrantes Digitales. 1-23. Obtenido de https://marcprensky.com/writing/Prensky-NATIVOS\%20E\%20INMIGRANTES\%20DIGITALES\%20(SEK).pdf

Restrepo, B. (2004). La investigación-acción-educativa y la construcción del saber pedagógico. Opción y Educadores(7), 45-55. Obtenido de https://www.redalyc.org/pdf/834/83400706.pdf

Sánchez, E., e Inzunza, S. (2006). Meanings' construction about sampling distributions in a dynamic statistics environment. En A. Rossman y B. Chance. En I. A. Education, A. Rossman, \& B. Chance (Edits.), ICOTS 7 
(págs. 1-6). Bahía: Education, International Association for Statistical. Obtenido de https://www.stat.auckland.ac.nz/ iase/publications/17/7C3_SANC.pdf

Trigueros, J., Sanchez, R., y Vera, M. I. (2012). El profesorado de Educación Primaria ante las TIC: realidad y retos. Revista electrónica interuniversitaria de formación del profesorado, 15(1), 101-112. Obtenido de https://dialnet.unirioja.es/servlet/articulo?codigo $=4616907$

UNESCO. (2008). Estándares TIC para la formación inicial docente. Unesco. Obtenido de https://www.campuseducacion.com/blog/wpcontent/uploads/2017/02/Normas_UNESCO_sobre_Competencias_en_TIC_para_Docentes.pdf

Uribe, A. (2015). Diseño de una propuesta curricular para docentes de la básica primaria, enfocada a la integración de las tecnologías de la información y las comunicaciones (tic) en el modelo escuela nueva escuela activa, en la Institución Educativa Rural (IER). Universidad Pontificia Bolivariana. Obtenido de https://repository.upb.edu.co/handle/20.500.11912/2838

Esta obra está bajo una Licencia Creative Commons Attribución-NoCommercial 4.0 International

(cc) EY-NC 